\title{
Empörung ist der Partner der Kommunikation
}

Kommunikation ist heute auch aus Verbänden nicht mehr wegzudenken. Denn deren Wahrnehmung - sowohl gegen innen als auch gegen aussen - und damit deren Erfolg sind wesentlich durch die Einstellung und das Verhalten der Stakeholder geprägt. Weil Kommunikation zunehmend komplexer wird und «Sprechen» oder «Schreiben» allein nicht mehr ausreicht, lud die FMH die Kommunikationsverantwortlichen der in der Ärztekammer vertretenen Organisationen zum ersten Treffen unter dem Motto «Lunch \& Learn» ein. Insgesamt 28 Gäste folgten der Einladung nach Bern und nutzten das Treffen als Informations- und Austauschmöglichkeit. Die Veranstaltung stiess auf viel positives Echo und wird zukünftig viermal jährlich durchgeführt.

Kommunikation ist auch das Handwerk von Dr. Oswald Sigg, Bundesratssprecher und Vizekanzler. Er koordiniert als oberste Kommunikationsinstanz Inhalte sowie deren Gewichtung und Timing - von insgesamt rund 500 Personen und 250 Kommunikationsstellen, so viele sind es nämlich, nimmt man alle Departemente und Bundesämter zusammen. Ein Grossteil der kommunikativen Aktivitäten ist auf die Medien bzw. deren Journalisten ausgerichtet. Nicht von ungefähr bezeichnet man diese deshalb als die vierte Gewalt im Staat. Die öffentliche Meinung ist wesentlich durch die Medien in der Rolle von Multiplikatoren geprägt, und gerade bei Urnenentscheiden, so Sigg, seien die Medien die Bühne der Politik. Darum gilt es, den Journalisten Sorge zu tragen und sie zu pflegen. Das Leitbild der Konferenz der Informationsdienste der Departemente und Bundesämter enthält darum auch den Grundsatz «Wir informieren und kommunizieren frühzeitig, sachlich und wahr, umfassend, kontinuierlich und transparent».

Genügt dieser Grundsatz noch in einer Zeit der zunehmenden Skandalisierung und Personalisierung von Nachrichten? Unabhängiger Journalismus ist auch in der Schweiz zur Rarität geworden; längst hat der Kommerz Einzug gehalten und beeinflusst die Chefredaktionen. Primeur-Geschichten sind gefragter denn je, denn auch Journalistinnen und Journalisten müssen ihre Geschichten ihrem Medium verkaufen, wenn sie im harten Wettbewerb bestehen wollen. Was also ist der entscheidende Faktor, dass eine Mitteilung einer Organisation von den Medienschaffenden aufgegriffen wird? Das Rezept ist einfach und gemäss Sigg lässt es sich mit einem Wort bezeichnen: Empörung. In der täglichen Informationsflut erregt eine Medienmitteilung dann das Interesse eines Journalisten, wenn sie empört, wenn sie also durch einen genügend starken Reiz die Aufmerksamkeit des Lesenden bewirkt und bei diesem Empörung auslöst.

Empörung ist also das Erfolgsrezept für die Aufmerksamkeit von Journalisten und damit der Schlüssel zum Betreten der Medienarena. Den Reiz anzustossen für die folgende Kettenreaktion ist sicherlich ein anzustrebendes Ziel im Umgang mit Journalisten. So werden wohl manche der Anwesenden zukünftig beim Schreiben einer Medienmitteilung oder im Vorfeld eines persönlichen Kontaktes mit einem Journalisten überlegen, wie sie ihre Kommunikation wohl mit einem Reiz anreichern können, der schliesslich Empörung beim Journalisten auslöst.

Empören, um Aufmerksamkeit zu erregen - das muss nicht sensationslüsterne und boulevardeske Kommunikation sein. Denn die kommunikativen Grundsätze aus Bundesbern - frühzeitig, umfassend, kontinuierlich informieren und kommunizieren und dabei sachlich, wahr und transparent bleiben - lassen sich auch mit der von Sigg dargestellten Empörungskommunikation vereinbaren. Manchmal reicht bereits ein Perspektivenwechsel: Warum nicht einmal die Brille der Medienkonsumenten aufsetzen und sich überlegen, welcher Aspekt diese an einer (noch zu schreibenden) Medienmitteilung speziell interessieren würde und sie in ihrem Alltag betreffen könnte? Je nach Perspektive wird dann eine Mitteilung z.B. über die Einschränkung der freien Arztwahl mehr oder eben weniger «empörend» ausfallen.

Vielfach können wir auch von der Empörungskommunikation anderer Keyplayer im Gesundheitswesen profitieren. Nicht selten kommt es vor, dass unsere Stimme zu einem bereits von den Krankenkassen oder dem Apothekerverband lancierten Thema noch gewünscht wird. Spätestens an diesem Punkt wird man sich dann wieder einmal bewusst, dass frühzeitige Kommunikation für einen Verband mit basisdemokratischer Ausrichtung und daher manchmal langen Vernehmlassungswegen mindestens ebenso herausfordernd ist wie Empörungswirtschaft. Vor allem auch im Hinblick auf die gesundheitspolitischen Themen der Wintersession, die u.a. die freie Arztwahl, eine für alle zugängliche und qualitativ hochstehende Gesundheitsversorgung sowie die ärztliche Weiterbildung in Frage stellen. Und die darum Bestandteil der kommunikativen Agenda der FMH sind.

Dr. med. Jacques de Haller, Präsident der FMH 